(C) 2017 IEEE. Personal use of this material is permitted. Permission from IEEE must be obtained for all other uses, in any current or future media, including reprinting/republishing this material for advertising or promotional purposes, creating new collective works, for resale or redistribution to servers or lists, or reuse of any copyrighted component of this work in other works. 


\title{
Opportunistic Access to PAL Channel for Multi-RAT GAA Transmission in Spectrum Access System
}

\author{
Shubhekshya Basnet*, Beeshanga Aberwardana Jayawickrama*, \\ Ying $\mathrm{He}^{*}$, Eryk Dutkiewicz*, Markus Dominik Mueck ${ }^{\dagger}$ \\ * University of Technology Sydney, Global Big Data Technologies Centre, Australia. \\ $\dagger$ Intel Mobile Communications, Germany. \\ Email: \{shubhekshya.basnet,ying.he-6\}@ student.uts.edu.au, \\ \{eryk.dutkiewicz, beeshanga.jayawickrama\}@uts.edu.au, markus.dominik.mueck@intel.com
}

\begin{abstract}
Spectrum Access System (SAS) is a three tier spectrum sharing framework proposed by the FCC. In this framework the aggregate interference of tier-3 General Authorised Access (GAA) users should be below a predetermined threshold anywhere within the tier-2 Priority Access Licensee (PAL) exclusion zone. GAA are expected to use a diverse range of Radio Access Technologies (RATs) with different levels of loading. We propose an optimal GAA power allocation and probability of spectrum utilisation scheme that meets the average aggregate interference constraint within the GAA network. Most of the capacity maximisation studies consider the instantaneous aggregated interference from secondary users. In this paper we present an average aggregated interference method to optimise the capacity of GAA users in a single channel. Simulation results suggest that we can significantly increase the capacity of the channel by considering the probability spectrum utilisation of GAA users.
\end{abstract}

Keywords-SAS, Interference Mitigation, Probability of Spectrum Utilisation

\section{INTRODUCTION}

Spectrum demand in wireless communications is increasing rapidly and more spectrum resources are required to meet this demand. Spectrum Access System (SAS) is a three tier system proposed by FCC for managing the frequency band 3550 to $3700 \mathrm{MHz}(3.5 \mathrm{GHz})$ for Citizen Broadband Radio Service (CBRS). CBRS is an opportunity to maximise the network capacity by spectrum sharing. CBRS consists of PAL and GAA users and both are assigned frequency resource at given locations by SAS [1]-[3]. PAL and GAA users are authorised by SAS for a finite census tract. A census tract is defined as a statistical subdivision of a country or equivalent entity. In SAS system, a PAL receives interference protection from GAA users and a GAA user receives no protection from Incumbent Access (IA) and Citizen Broadband Radio Service Devices (CBSDs) which includes PAL and other GAA users.

Fig. 1 shows the graphical representation of frequency arrangement for $3.5 \mathrm{GHz}$ band. PAL will be assigned $70 \mathrm{MHz}$ of $3.5 \mathrm{GHz}$ band and GAA will be allowed throughout the $150 \mathrm{MHz}$ band. Each PAL channel is of $10 \mathrm{MHz}$ bandwidth

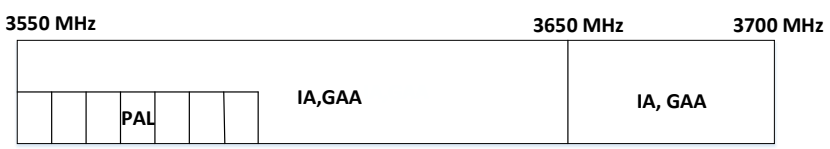

Fig. 1: $3.5 \mathrm{GHz}$ Band Plan

[2]. In this study, we consider one single PAL channel which is utilised opportunistically by GAA users when it is not utilised by the PAL user.

PAL users must report their PAL protection area to SAS depending upon their network deployment. PAL protection criteria as defined in [2] is:

"To ensure that Priority Access operations are protected from harmful interference, an aggregate received signal level at PAL license boundaries to be at or below an average power level of $-80 \mathrm{dBm}$ when integrated over a $10 \mathrm{MHz}$ reference bandwidth with the measurement antenna placed at a height of 1.5 meters above ground level."

Several studies have already been performed on the cognitive radio and dynamic spectrum access to maximise the spectrum efficiency by controlling the interference from secondary users (SU) to primary users (PU) [4]-[7] . However, all of these studies are based on the instantaneous interference from SU to PU without considering the duty cycle of secondary users. In [8] with prior information regarding the PU idle period, authors formulate an optimisation problem to maximise the use of spectrum holes under the constraint of probability of collision. The aggregate interference caused by secondary users was not considered in this study. For optimal power allocation in cognitive radio network, in [9] authors considered the transmit power and instantaneous interference constraints. However, the transmit power allocation did not consider the transmit time of secondary users.

Hetereogeneous networks consist of various networks using different Radio Access Technologies (RATs) and has attracted consideration to minimise the spectrum scarcity issue by increasing data rate and throughput in unlicensed 
spectrum [10]. According to [11] networks will be more heterogeneous as we transition to $5 \mathrm{G}$ and there will be more combinations among various RATs and the probability of occupying the wireless medium (will be referred to as spectrum utilisation here onwards) varies between different RATs. This work focus on maximising the capacity for the opportunistic spectrum access to PAL channels for multiRAT GAA transmission.

The main contributions of this study can be summarized as follows:

- To the best of our knowledge, for the first time the optimal transmit power allocation problem is investigated for GAA users considering the probability of spectrum utilisation of CBSDs in SAS system.

- Optimal probability of spectrum utilisation and transmit power allocation are obtained such that sum capacity of GAA network is maximised.

- To increase the capacity of GAA users, we consider the average aggregated interference from GAA users and the probability of spectrum utilisation of each GAA user.

- Our approach shows that more power is allocated to GAA which transmit for less time and less power to the one which transmit for more, and the probability of spectrum utilisation increases with distance.

The rest of the paper is organized in five sections. Section II introduces the system model and presents the assumptions. In section III, we formulate the optimisation problem. The numerical results from the optimisation problem are given in section IV. Finally, conclusions are shown in section V.

\section{SYSTEM MODEL}

In this study we consider the scenario where one PAL user within a census tract is sharing a PAL channel with neighbouring GAAs within the same census tract. GAA users are randomly located in space. In the current SAS architecture each PAL and GAA should always report the transmission characteristics such as the power, antenna radiation pattern and the location to the SAS [1], [2]. We consider a scenario where both PAL and GAA report to the same SAS. Therefore, the SAS is aware of the transmission characteristics including the locations of all PAL and GAA users.

The GAAs could be a heterogeneous network that uses different RATs. Further the GAAs would have different network loads. Hence some GAA networks are expected to be more active than others. In our work we define the probability of spectrum utilisation as the fraction of time in which a GAA user is active in a certain time period.

The probability of spectrum utilisation of the $i$ th GAA user $\left(u_{i}\right)$ is given by:

$$
u_{i}=\lim _{N_{t} \rightarrow \infty} \frac{t_{o}}{N_{t}}
$$

where, $t_{o}$ is the transmitting time if the channel was exclusive to $i$ th GAA and $N_{t}$ is the total time period. The $t_{0}$ depends on the RAT and number of end users in the $i$ th GAA network.

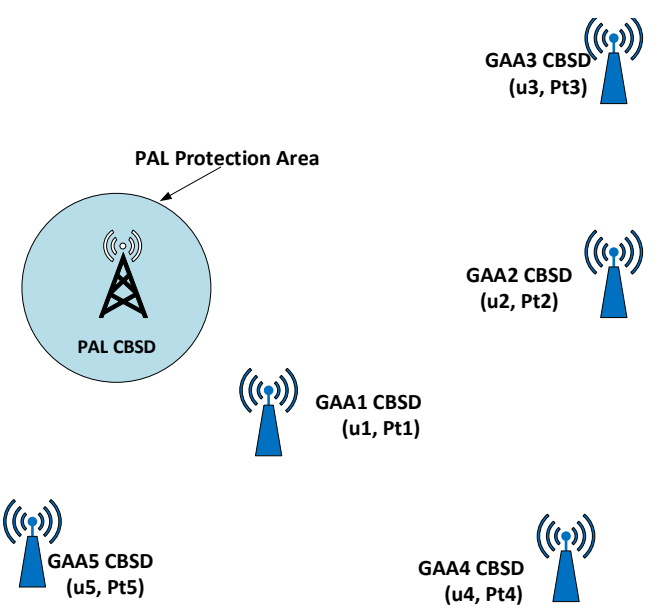

Fig. 2: Single PAL and Multiple GAA

In SAS system GAA users can operate in priority access channel opportunistically in a non interfering way. Other PAL or GAA CBSD's will not be authorized by SAS to operate on the same channel with aggregate interference above the interference threshold within PAL protection area i.e.

$$
I_{\text {agg }} \leq I_{t h}
$$

where, $I_{a g g}$ is the aggregate interference inside the PAL protection area and $I_{t h}$ is the interference threshold.

\section{Transmit Power and Probability of Spectrum UTILISATION OPTIMISATION FOR MAXIMISATION OF AVERAGE CAPACITY}

In this section we propose the capacity maximisation for GAA users by controlling the transmit power of GAA users considering different probability of spectrum utilisation in a multi-RAT environment. Our objective is to find the maximum transmit power and probability of spectrum utilisation allocation for GAA users such that the sum capacity of GAA network is maximised while maintainting the average aggregate interference from GAA users is below the interference threshold.

\section{A. Capacity}

PAL and GAA should provide their geo-location information to SAS system. Winner II Path Loss (PL) model is used to calculate PL between GAA and PAL CBSD, and is given by [12]:

$$
P L_{i}(d B)=46.4+20 \times \log _{10} d_{i}+20 \times \log _{10} \frac{f}{5.0}
$$

where, $d_{i}$ is distance between PAL and $i$ th GAA CBSD's in meters and $f$ is the frequency in $\mathrm{GHz}$.

We consider the downlink transmission of GAA users. The received power of the $k$ th, $k=1,2, \ldots, N_{U E}$ GAA receiver 
from the $i$ th GAA user is given by:

$$
P r^{i, k}=\frac{P t_{i}}{P L_{i, k}}
$$

where $P t_{i}$ is the transmit power of $i$ th GAA basestation, $P L_{i, k}$ is the pathloss between $i$ th GAA basestation and $k$ th GAA end user.

The instantantaneous downlink capacity for $i$ th GAA network at the time $t$ is given by:

$$
C_{i}(t)=\sum_{k=1}^{N_{U E}^{i}} \log \left(1+\frac{P_{r}^{i, k}}{P_{N}+\sum_{b \in\left\{S_{I}^{i}\right\} \backslash\{i\}} P_{r}^{b, k}}\right)
$$

where, $N_{U E}$ is total number of GAA user equipment and $S_{I}{ }^{i}$ is subset of active GAAs when $i$ th GAA is active. $\sum_{b \in\left\{S_{I}^{i}\right\} \backslash\{i\}} P_{r}^{b, k}$ is the received power by UEs from GAAs active subset.

It is assumed that GAA users are sufficiently apart when transmitting and have different probability of spectrum utilisation. Probability of $S_{I}$ subset doing the transmission is given by:

$$
P_{S_{I}}=\prod_{b \in\left\{S_{I}^{i}\right\}} u_{b} \prod_{m \in\left\{S_{N}\right\} \backslash\left\{S_{I}^{i}\right\}}\left(1-u_{m}\right)
$$

where, $u_{m}$ is the probability of spectrum utilisation of inactive GAAs, $u_{b}$ is the probability of spectrum utilisation of the active GAAs and $S_{N}$ is a set of all GAA users.

Average capacity when $S_{I}$ set of users are transmitting is given by:

$$
C_{S_{I}}=P_{S_{I}} \sum_{k=1}^{N_{U E}^{i}} \log \left(1+\frac{P_{r}^{i, k}}{P_{N}+\sum_{b \in\left\{S_{I}^{i}\right\} \backslash\{i\}} P_{r}^{b, k}}\right)
$$

where, $P_{r}^{i, k}$ is the received power by $k$ th UE from transmitting GAA i.e. $i$ th GAA, $P_{S_{I}}$ is from equation (6)

The average downlink capacity when $i$ th GAA is transmitting considering different combination of $S_{I}$ is given by equation (8).

$$
\begin{aligned}
C_{i}= & \sum^{\left\{S_{I}^{i}\right\} \in\left\{S_{S}^{i}\right\}}\left(\prod_{b \in\left\{S_{I}^{i}\right\}} u_{b} \prod_{m \in\left\{S_{N}\right\} \backslash\left\{S_{I}^{i}\right\}}\left(1-u_{m}\right)\right. \\
& \left.\sum_{k=1}^{N_{U E}^{i}} \log \left(1+\frac{P_{r}^{i, k}}{P_{N}+\sum_{b \in\left\{S_{I}^{i}\right\} \backslash\{i\}} P_{r}^{b, k}}\right)\right)
\end{aligned}
$$

where, $S_{S}$ is all the possible combination of $S_{I}$ that includes $i$ th GAA.

In our work we assume GAA performs Listen Before Talk (LBT) to sense other CBSDs, and GAAs will not transmit in the same channel if they sense other PAL or GAA users. So, in our study we consider the probability of spectrum utilisation of only transmitting GAA user and do not consider the interference from other GAA users transmitting in the same channel. The average downlink capacity of GAA cell is given by:

$$
C_{G A A}=u_{i} \sum_{k=1}^{N_{U E}} \log _{2}\left(1+\frac{P_{r}^{i, k}}{P_{N}^{k}}\right)
$$

We consider $N_{G A A}$ number of GAAs transmits in PAL channel opportunistically over the time period $N_{t}$. The average aggregated downlink capacity of all GAA users is given by:

$$
C_{a g g}=\sum_{i=1}^{N_{G A A}} u_{i} \sum_{k=1}^{N_{U E}} \log _{2}\left(1+\frac{P_{r}^{i, k}}{P_{N}^{k}}\right)
$$

\section{B. Average Aggregate Interference}

The interference to PAL CBSD from $i$ th GAA user is given by:

$$
I_{i}=\frac{P t_{i}}{P L_{i, P A L}}
$$

where, $I_{i}$ is the interference from $i$ th GAA to PAL, $P t_{i}$ is the transmit power of $i$ th GAA and $P L_{i, P A L}$ is the path loss (as a ratio-not $\mathrm{dB}$ ) from $i$ th GAA user to PAL.

The instantantaneous aggregate interference from GAA users to PAL at the certain time is given by:

$$
I_{a g g}=\sum_{i=1}^{N_{G A A}} \frac{P t_{i}}{P L_{i, P A L}}
$$

With multi-RAT GAA users transmitting with different probability of spectrum utilisation, the average aggregate interference from all GAA users to PAL for time period $N_{t}$ is given by:

$$
I_{a v g}=\sum_{i=1}^{N_{G A A}} \frac{P t_{i}}{P L_{i, P A L}} \times u_{i}
$$

C. Convex Optimisation for transmit power allocation when probability of spectrum utilisation is known

In this section we extend the current SAS architecture by allowing GAAs to report the probability of spectrum utilisation as shown in equation (1) representing the activity level within its network. The optimisation problem is to find the optimal power allocation for GAAs that maximises the average sum capacity of GAA users subject to transmit power and average aggregated interference constraints. The optimization problem can be formulated as:

$$
\begin{array}{ll}
\text { maximize } & \sum_{i=1}^{N_{G A A}} \log _{2}\left(1+P t_{i}\right) \times u_{i} \\
\text { subject to } & 0<P t_{i}<P_{\max } \\
& I_{\text {avg }} \leq I_{t h}
\end{array}
$$

where, $P_{\max }$ is the maximum allowed transmit power for GAA users.

\section{Convex Optimisation for transmit power and probability of spectrum utilisation allocation}

The optimisation problem is to find the optimal power and probability of spectrum utlisation allocation for GAA 


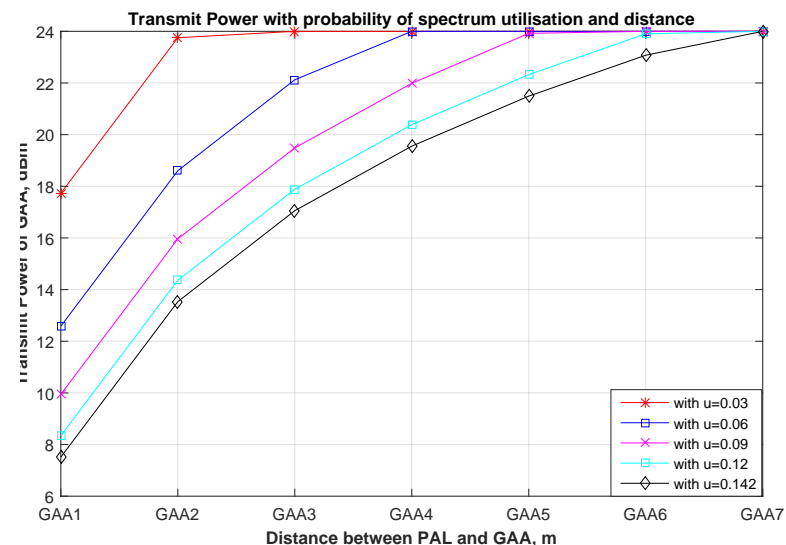

Fig. 3: Transmit Power of GAA users with different probability of spectrum utilisation, Results of Convex Optimisation for transmit power allocation when probability of spectrum utilisation is known

users. The average sum capacity of GAA users is maximised subject to transmit power, probability of spectrum utilisation of GAA users and average aggregated interference constraints. The sum of probability of spectrum utilisation of all GAA users should be less than or equal to 1 . Using this proposed method in current SAS architecture where CBSD's need to report their locations to SAS, GAA users can be allocated optimal transmit power and probability of spectrum utilisation. The optimization problem can be formulated as:

$$
\begin{array}{ll}
\text { maximize } & \sum_{i=1}^{N_{G A A}} \log _{2}\left(1+P t_{i}\right) \times u_{i} \\
\text { subject to } \quad & 0<P t_{i}<P_{\max } \\
& I_{a v g} \leq I_{t h} \\
& \sum_{i=1}^{N_{G A A}} u_{i} \leq 1
\end{array}
$$

where, $u_{i}$ is the probability of spectrum utilisation of the $i$ th GAA user.

\section{NUMERICAL RESULTS}

In this section we present simulations results for our optimisation problem which is presented in Section III. To solve our optimisation problem we used convex optimisation in MATLAB. We consider a scenario with single PAL and 7 GAA users at different locations sufficiently apart. For each GAA user we considered a cell of radius $40 \mathrm{~m}$ with 6 GAA UE at different geo-location within the cell. In our simulations we have set the $P_{\max }$ as $24 \mathrm{dBm}$ and $I_{t h}$ as -80 $\mathrm{dBm}$ based on FCC technical requirements for CBSD's [2].

Fig. 3 shows the transmit power allocation to GAA CBSD's based on our optimisation results. We considered different scenarios with different probability of spectrum utilisation, in which GAA users are transmitting with same probability of spectrum utilisations. Results shows that greater the probability of spectrum utilisation, lower power the GAA gets which alligns with the target of our method. Results also shows that the distance more than $350 \mathrm{~m}$ from PAL can transmit with maximum transmit power with any probability of spectrum utilisation.

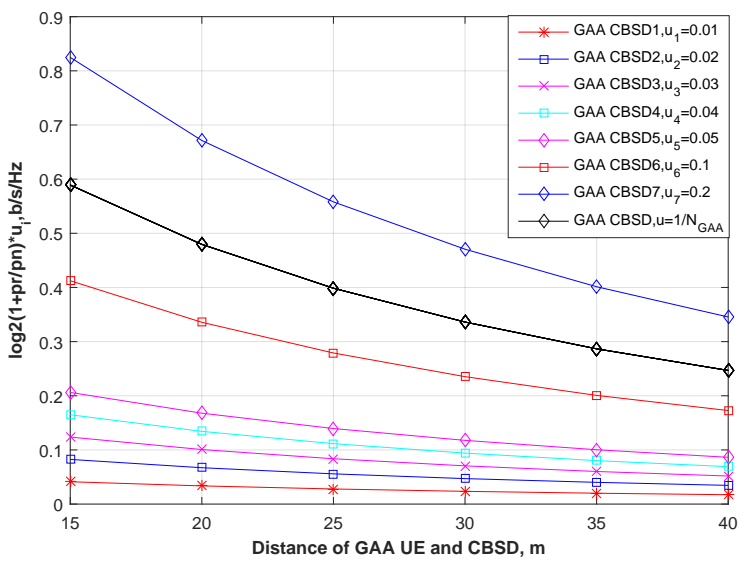

Fig. 4: Downlink Capacity of GAA users

Fig. 4 shows the capacity of GAA receiver at different probability of spectrum utilisation and distance for different GAA CBSD. GAA users have different transmission probability with average probability of spectrum utlisation less than one. It is seen from the results that the average capacity can be maximised by controlling probability of spectrum utilisation of GAA users. The black plot shows the average capacity when the channel is fully used for the time period i.e. all GAA users are transmitting with same probability of spectrum utilisation.

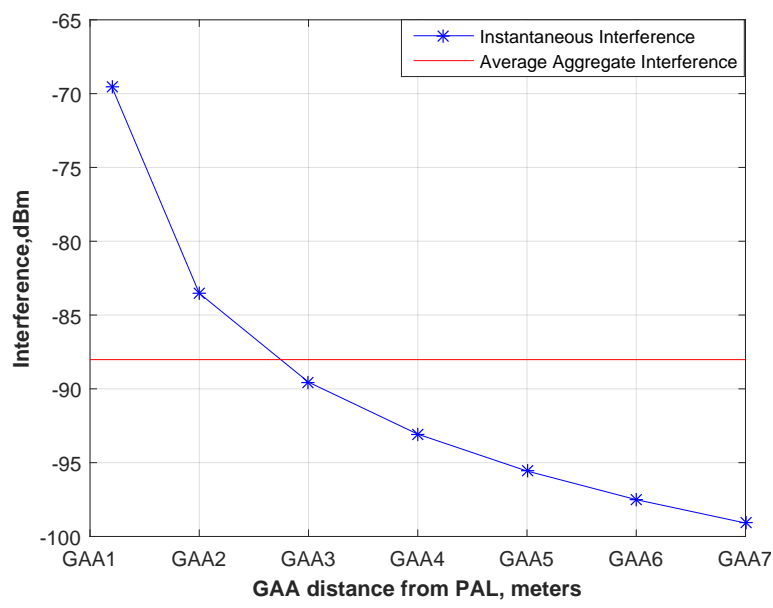

Fig. 5: Instantaneous and average aggregated interference to PAL from GAA users at different distance

Fig. 5 shows the instantaneous and average aggregated interference from GAA users at different distance from PAL. Average aggregate interference considers the probability of spectrum utilisation of GAA users and is $-88.1 \mathrm{dBm}$ which is less than maximum allowed interference threshold, 


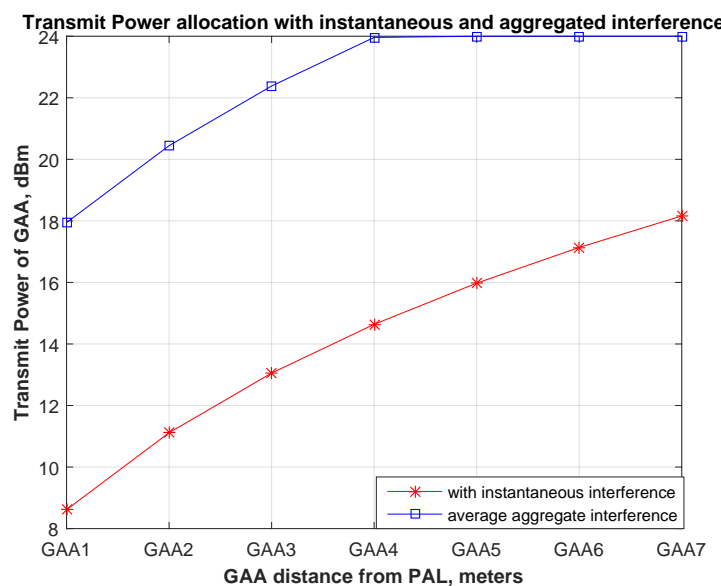

Fig. 6: Transmit power allocation for GAA users, Results of Convex Optimisation for transmit power and probability of spectrum utilisation allocation

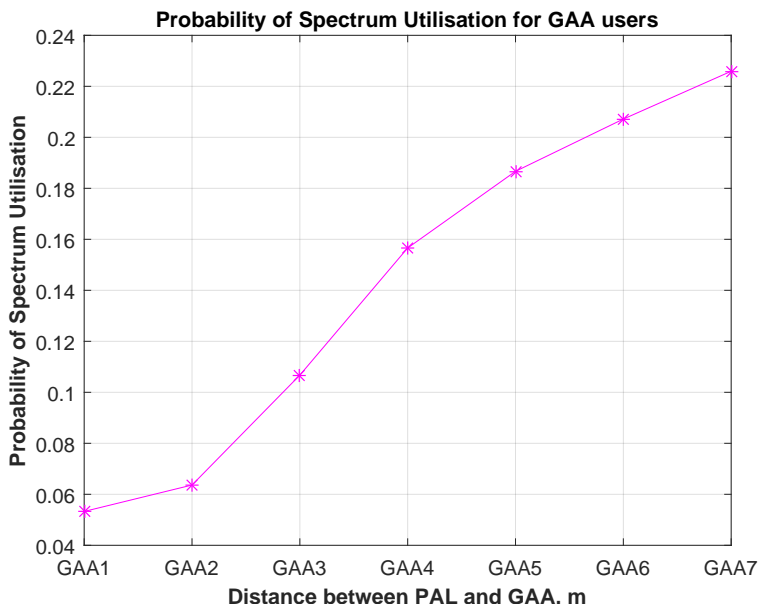

Fig. 7: Probability of spectrum utilisation allocation for GAA users, Results of Convex Optimisation for transmit power and probability of spectrum utilisation allocation

whereas the instantaneous interference do not consider probability of spectrum utilisation i.e. probability of spectrum utilisation is 1 for all GAA users.

Fig. 6 shows the optimisation results for transmit power allocation for GAA which are transmitting at different distances from PAL when considering the constraint as instantaneous interference and average aggregated interference. Results from optimisation shows that we can maximise, the transmit power allocation for GAA users by considering the probability of spectrum utilisation of GAA users.

Fig. 7 shows the probability of spectrum utilisation allocation for GAA users. Results from optimisation shows that the probability of spectrum utilisation for GAA users increases with distance.

\section{CONClusion}

In this paper, the optimal transmit power and probability of spectrum utilisation has been investigated for the sum capacity maximisation of GAA network. First, we propose an optimum power allocation scheme for multi-RAT GAA users taking into consideration that the probability of occupying the medium differs across GAAs using different RATs. Then, the optimal transmit power and probability of spectrum utilisation is allocated for GAA users when only locations of PAL and GAA users are known. We maximise the average capacity of the GAA network while the average aggregate interference to the PAL exclusion zone is kept below a predetermined threshold. We compare our approach of average aggregate interference considering the probability of spectrum utilisation with the instantaneous aggregate interference without considering the probability of medium occupancy of different RATs. Transmit power allocation to GAA users can be maximised by 8 to $10 \mathrm{dBm}$ when considering the average aggregate interference and the average aggregate interference is $-88.1 \mathrm{dBm}$ which is much less than maximum allowable interference threshold i.e. -80 $\mathrm{dBm}$. Numerical results shows that our approach is effective in maximising the capacity of GAA users by taking into consideration the probability of spectrum utilisation of GAA.

\section{ACKNOWLEDGEMENTS}

This work has been supported by Intel's University Research Office.

\section{REFERENCES}

[1] Report and Order and Second Further Notice of Proposed Rulemaking. Federal Communications Commision, 2015.

[2] Order on reconsideration and second report and order. Federal Communications Commision, 2016.

[3] M. M. Sohul, M. Yao, T. Yang, J. H. Reed, Spectrum Access System for the Citizen Broadband Radio Service. IEEE Communications Magazine, 2015.

[4] S. Singh, P. Teal, P. Dmochowski, A. Coulson, Interference Management in Cognitive Radio Systems - a convex optimisation approach IEEE ICC 2012- Cognitive Radio and Networks Symposium, 2012.

[5] S. Kusaladharma, P. Hearth, C. Tellambura, Impact of Transmit Power Control on Aggregate Interference in Underlay Cognitive Radio Networks. IEEE International Conference on Communication, 2014.

[6] T. Zhang, L. An, Y. Chen, K. Chai, Aggregate Interference Statistical Modeling and User outage Analysis of Heterogeneous Cellular Networks. IEEE ICC-Communication QoS, Reliability and Modeling Symposium, 2014.

[7] L. Vijayandran, P. Dharmawansa, T. Ekman, C. Tellambura, Analysis of Aggregate Interference and Primary System Performance in Finite Area Cognitive Radio Networks. IEEE transaction on Communication, 2012.

[8] Q. Xiao, Q. Gao, L. Xiao, S. Zhou, J. Wang, An Optimal Opportunistic Spectrum Access Approach. IEEE International Conference on Communications Workshop, 2009.

[9] X. Kang, Y. Liang, A. Nallanathan, Optimal Power Allocation for Fading Channels in Cognitive Radio Networks under Transmit and Interference Power Constraints. IEEE International Conference on Communications, 2008.

[10] J. Li and Y. Han, Multi-RAT Wireless Network Capacity Optimization under Optimal Spectrum Splitting in LTE-U. IEEE Wireless Communications and Networkinf Conference, 2016.

[11] J. G. Andrews, S. Buzzi, W. Choi, S. V. Hanly, A. Lozano, A. C. K. Soong, and J. C. Zhang, What will 5G be?IEEE Journal on Selected Areas in Communications, vol. 32, no. 6, pp. 10651082, June 2014.

[12] P. kyostii, J. Meinila, L. Hentila, X. Zhao, T. Jamsa, C. schneider, M. Narandzic, M. Milojevic, A. Hong, J. Ylitalo, V. Hollappa, M. Alatossava, R. Bultitude, Y. Jong, T. Rautiainen, Winner II channel models.Information Society Technologies, 2008. 